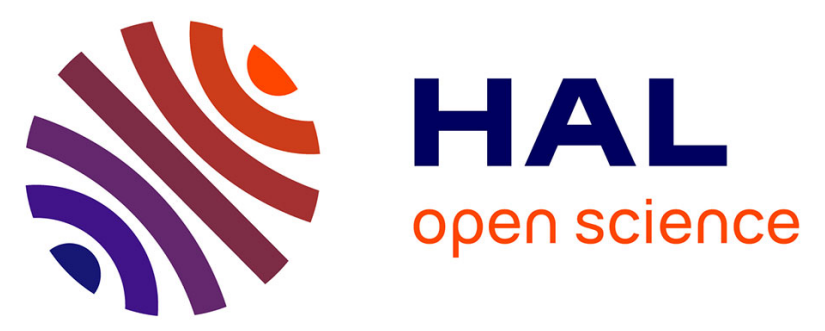

\title{
Sorting of Leishmania-bearing dendritic cells reveals subtle parasite-induced modulation of host-cell gene expression
}

Hervé Lecoeur, Emilie de La Llave, José Osorio y Fortéa, Sophie Goyard, Helene Kiefer-Biasizzo, Anne-Marie Balazuc, Geneviève Milon, Eric Prina, Thierry Lang

\section{To cite this version:}

Hervé Lecoeur, Emilie de La Llave, José Osorio y Fortéa, Sophie Goyard, Helene Kiefer-Biasizzo, et al.. Sorting of Leishmania-bearing dendritic cells reveals subtle parasite-induced modulation of hostcell gene expression. Microbes and Infection, 2010, 12 (1), pp.46-54. 10.1016/j.micinf.2009.09.014 . pasteur-02794034

\section{HAL Id: pasteur-02794034}

\section{https://hal-pasteur.archives-ouvertes.fr/pasteur-02794034}

Submitted on 16 Jun 2020

HAL is a multi-disciplinary open access archive for the deposit and dissemination of scientific research documents, whether they are published or not. The documents may come from teaching and research institutions in France or abroad, or from public or private research centers.
L'archive ouverte pluridisciplinaire HAL, est destinée au dépôt et à la diffusion de documents scientifiques de niveau recherche, publiés ou non, émanant des établissements d'enseignement et de recherche français ou étrangers, des laboratoires publics ou privés. 


\title{
Sorting of Leishmania-bearing dendritic cells reveals subtle parasite-induced modulation of host-cell gene expression ${ }^{\text {it }}$
}

\author{
Hervé Lecoeur ${ }^{\text {a }}$, Emilie de La Llave ${ }^{\text {a }}$, José Osorio Y Fortéa ${ }^{\mathrm{a}}$, Sophie Goyard ${ }^{\mathrm{a}}$, \\ Hélène Kiefer-Biasizzo ${ }^{b}$, Anne-Marie Balazuc ${ }^{b}$, Geneviève Milon ${ }^{a}$, \\ Eric Prina ${ }^{\mathrm{a}, 1}$, Thierry Lang ${ }^{\mathrm{a}, *, 1}$ \\ ${ }^{a}$ Institut Pasteur, Unité d'Immunophysiologie et Parasitisme Intracellulaire, Département de Parasitologie et Mycologie, 25 rue du Dr. Roux, \\ 75724 Paris Cedex 15, France \\ ${ }^{\mathrm{b}}$ Institut Pasteur, Plateforme de Cytométrie en Flux, 25 rue du Dr. Roux, 75724 Paris cedex 15, France
}

Received 17 July 2009; accepted 20 September 2009

Available online 26 September 2009

\begin{abstract}
Once in the mouse skin, Leishmania (L) amazonensis amastigotes are hosted by professional mononuclear phagocytes such as dendritic cells (DCs). When monitored after parasite inoculation, the frequency of amastigote-hosting DCs is very low $(<1 \%)$ in both the skin and skindraining lymph nodes. Therefore, we designed and validated an efficient procedure to purify live amastigotes-hosting DCs with the objective to facilitate quantitative and qualitative analysis of such rare cells. To this end, a L. amazonensis transgenic parasite expressing $D s R e d 2$ fluorescent protein was generated and added to mouse bone marrow-derived DC cultures. Then, a high speed sorting procedure, performed in BSL-2 containment, was setup to pick out only DCs hosting live amastigotes. This study reveals, for the first time, a unique transcript pattern from sorted live amastigotes-hosting DCs that would have been undetectable in unsorted samples. It was indeed possible to highlight a significant and coordinated up-regulation of L-arginine transporter and arginase2 transcripts in Leishmania-hosting DCs compared to un-parasitized DCs. These results indicate that arginine catabolism for polyamine generation is dominating over L-arginine catabolism for NO generation. In conclusion, this approach provides a powerful method for further characterisation, of amastigote-hosting DCs in the skin and the skin-draining lymph nodes. (C) 2009 Elsevier Masson SAS. All rights reserved.
\end{abstract}

Keywords: Leishmania; Mouse dendritic cells; Flow-sorting; BSL-2; DsRed2

\section{Introduction}

Leishmania $(L)$ amazonensis is a protozoan parasite that is transmitted to the dermis of its mammalian host by blood-

\footnotetext{
it Conceived and designed the study : HL, GM, EP, TL. Performed the experiments HL, ELL, HKB, EP, TL. Contributed reagents / materials/ analysis tool: JOYF, SG, AMB. Wrote the paper: HL, GM, EP, TL.

* Corresponding author.

E-mail addresses: herve.lecoeur@pasteur.fr (H. Lecoeur), emilie.de-lallave@pasteur.fr (E. de La Llave), Jose.OSORIOYFORTEA@limagrain.com (J. Osorio Y Fortéa), sophie.goyard@pasteur.fr (S. Goyard), helene.kieferbiasizzo@pasteur.fr (H. Kiefer-Biasizzo), anne-marie.balazuc@pasteur.fr (A.-M. Balazuc), genevieve.milon@pasteur.fr (G. Milon), eric.prina@ pasteur.fr (E. Prina), thierry.lang@pasteur.fr (T. Lang).

${ }^{1} \mathrm{EP}$ and TL contributed equally to this work.
}

feeding sand flies. Laboratory mice have been shown to be relevant hosts of this Leishmania species for in vivo and ex vivo analyses. Once in the dermis of the mouse, the metacyclic promastigotes enter dendritic cells (DCs) [1], where they differentiate into amastigotes within the parasitophorous vacuole. The stepwise deployment of both the migratory and signalling properties of DCs hosting Leishmania amastigotes within the tissues is still being investigated [2-5]. Thus, it is important to characterise the features of live amastigote-harbouring DCs sorted under the most rigorous conditions. Bone marrow-derived DCs cultured in the presence of GM-CSF have been shown to host L. amazonensis amastigotes $[1,6]$. However, the heterogeneity of this DC population [7,8], as well as the low frequency $(\leq 12 \%)$ of amastigote-loaded DCs, have prevented us from addressing questions about the range 
of functions these infected DCs could display. Therefore, to overcome these limitations, we designed and validated a reliable protocol to purify live Leishmania-hosting DCs. The present study describes the different steps of the procedure, from the generation of transgenic L. amazonensis expressing the fluorescent $D s R e d 2$ molecule (DsRed2-LV79) [9] to the optimal use of the FACSAria cell sorter in biosafety level 2 (BSL-2) containment [10,11].

\section{Materials and methods}

\subsection{Mice}

Female BALB/c mice and Swiss nu/nu mice were purchased from Charles River (Saint Germain-sur-l'Arbresle, France) and were housed following institutional guidelines of the A3 animal facility at the Institut Pasteur (Paris, France); http://webcampus.pasteur.fr/jcms/c_87141/documents.

\subsection{Generation of DsRed2-transgenic L. amazonensis}

The 697-bp DsRed 2 coding region was cut by BamHI/NotI from $\mathrm{p} D$ sRed2 (Clontech, CA) and subsequently cloned into Leishmania expression vector pF4X1.HYG (Jenabioscience, Jena, Germany) previously cut by BglII/NotI. This plasmid contains the Hygromycin B marker for the selection of transgenic Leishmania. In this construct, the 3' and 5' UTR regions flanking the DsRed2 and Hyg genes provide the required splicing and polyadenylation signals. Following linearization with SwaI, transfections were performed by electroporation in a $4 \mathrm{~mm}$ cuvette under the following conditions: 25 [rId7]F, $1500 \mathrm{~V}, 3.75 \mathrm{kV} / \mathrm{cm}$ [12]. Cells were incubated for $24 \mathrm{~h}$ in medium without the drug, and plated for selection of transgenic Leishmania on semi-solid medium containing $100 \mu \mathrm{g} / \mathrm{ml}$ of hygromycin B (Cayla, Toulouse, France). Recombination of the engineered plasmids leads to integration into the Leishmania rDNA locus, ensuring a permanent high level of transcription.

\subsection{L. amazonensis metacyclic promastigote preparation and inoculation into nude mice}

Wild type or DsRed2-transgenic L. amazonensis strain LV79 (WHO number MPRO/BR/72/M1841) amastigotes were isolated from Swiss nude mice inoculated 2 months before under a BSL-2 cabinet [1]. These amastigotes did not present antibodies at their surface $[1,13]$. Promastigotes derived from amastigotes were cultured at $26{ }^{\circ} \mathrm{C}$ in complete M199 medium [12]. Infective-stage metacyclic promastigotes were isolated from stationary phase cultures (6 day-old) on a Ficoll gradient [14]. Ten thousand metacyclic promastigotes per $10 \mu \mathrm{l}$ of PBS were injected into the ear dermis of BALB/c mice. Lesion size was measured using a direct reading Vernier caliper (Thomas) and expressed as ear thickness.

\subsection{Real-time cytofluorometric analyses of DsRed2- LV79 amastigotes}

Amastigote size was assessed with the forward scatter photodiode using the E-00 setting with a logarithmic amplification gain of 1.32. Amastigote structure was evaluated by the side scatter (SSC) photomultiplier tube, using a voltage of $363 \mathrm{~V}$ and a linear amplification gain adjusted to 6.88. DsRed 2 fluorescence was analysed in logarithmic scale. Real-time experiments were performed on samples containing $10^{6}$ parasites per $\mathrm{ml}$ in $3 \mathrm{ml}$ of M199 medium. Basal FSC, SSC and DsRed2 fluorescence data were acquired for $2 \mathrm{~min}$, and L-leucine methyl ester (Leu-oMe) or paraformaldehyde (PFA) were added at a final concentration of $2 \mathrm{mM}$ and $1 \%$, respectively. Data were collected for the following $14 \mathrm{~min}$ as previously described for isolated liver mitochondria [15]. FSC and $D s$ Red 2 fluorescence changes were analysed using the Flow Jo software (Ashland, OR).

\subsection{DsRed2-L. amazonensis amastigote addition to DC cultures}

DCs were differentiated from bone marrow cells of 6-weekold BALB/c mice according to a method adapted from Méderlé et al. $[1,16]$. Briefly, bone marrow cells were seeded at $2 \times 10^{6}$ cells per $100 \mathrm{~mm}$ diameter bacteriological grade Petri dish (Falcon, Becton Dickinson Labware, Franklin Lakes, NJ) in $10 \mathrm{ml}$ of Iscove's modified Dulbecco's medium (IMDM; BioWhittaker Europe, Verviers, Belgium) supplemented with $10 \%$ heat-inactivated foetal calf serum (FCS; Dutscher, Brumath, France), $1.5 \%$ supernatant from the GM-CSF producing J558 cell line [17], $50 \mathrm{U} / \mathrm{ml}$ penicillin, $50 \mu \mathrm{g} / \mathrm{ml}$ streptomycin, $50 \mu \mathrm{M} 2-$ mercaptoethanol and $2 \mathrm{mM}$ glutamine. Cultures were incubated at $37^{\circ} \mathrm{C}$ in a humidified atmosphere with $7 \% \mathrm{CO}_{2}$. On day 6 , suspended cells and loosely adherent cells were harvested using $1 \%$ EDTA (Versene) and cultured in complete IMDM supplemented with $10 \%$ of the primary culture supernatant. On day 10 , cells were harvested with EDTA and distributed in hydrophobic 6-well plates (Greiner, St Marcel, France) at a concentration of $9 \times 10^{5}$ cells $/ \mathrm{ml}$ in $3 \mathrm{ml}$ complete IMDM. On day 14 , DCs were exposed to freshly isolated DsRed2-LV79 amastigotes or to live BCG at micro-organism-DC ratios of 4:1 and 10:1, respectively. DC cultures were placed at $34{ }^{\circ} \mathrm{C}$ for $24 \mathrm{~h}$.

\subsection{Preparation of DC samples for cell sorting}

Five minutes post the incubation in Versen-EDTA at $34{ }^{\circ} \mathrm{C}$, DCs were carefully detached, resuspended at $4{ }^{\circ} \mathrm{C}$ in Dulbecco's PBS with 2\% FCS (PBS-FCS) and transferred to a $15 \mathrm{ml}$ tube (Falcon; BD Biosciences, San Jose, CA) at a concentration of $6 \times 10^{6}$ cells $/ \mathrm{ml}$. Cells were centrifuged $\left(300 \mathrm{~g}, 5 \mathrm{~min}, 4^{\circ} \mathrm{C}\right.$ ) and then incubated in PBS-FCS supplemented with $10 \%$ heat-inactivated donkey serum for $5 \mathrm{~min}$. Cells were incubated for $30 \mathrm{~min}$ in PBS-FCS containing $0.2 \mu \mathrm{g} / \mathrm{ml}$ of the anti-MHC class II monoclonal antibody (mAb) (M5/114) or the corresponding IgG2a isotype control $\mathrm{mAb}$, both conjugated to $\mathrm{PE}-\mathrm{Cy} 5$-conjugated $\mathrm{mAb}$ (eBioscience). After two washes, cells were resuspended at $5 \times 10^{6}$ 
cells $/ \mathrm{ml}$ in PBS containing 3\% FCS and 1\% J558 supernatant. Cell aggregates were dissociated on a $70 \mu \mathrm{m}$ filter (Falcon), and placed on ice until the cell sorting was done.

\subsection{Cell sorting and biosafety guidelines}

All experimental procedures were performed according to biosafety level two practises $[10,11]$. Biosafety guidelines can be obtained from one of us (HKB) (Plateforme de cytométrie en flux; Certification ISO 9001: 2000).

Cell sorting was performed using a FACSAria (BD Biosciences) equipped with completely sealed sample injection and sort collection chambers that operate under negative pressure. Following the M5/114 mAb staining, DCs were sorted using the BD FACSDiva software (BD Biosciences). PE-Cy5 and DsRed2 fluorescences were collected through $695 / 40$ and 576/26 bandpass filters respectively. FSC and SSC were displayed on a linear scale, and used to discard cell debris. To avoid the sorting of cell doublets or cell aggregates, single cells were sequentially selected on SSC-H/SSC-W, and FSC-H/FSC-W dot plots. Infected DCs were sorted by selecting cells expressing both surface MHC Class II molecules and DsRed2. Sorting conditions included-i) sheath pressure of $70 \mathrm{Psi}$, ii) flow rate of 7 and iii) $70 \mu \mathrm{m}$ nozzle tip. Cells were collected at $4{ }^{\circ} \mathrm{C}$ in polypropylene tubes (BD Biosciences) previously coated with FCS (1 night at $\left.4{ }^{\circ} \mathrm{C}\right)$. Sorted cells were immediately used for further studies.

\subsection{Detection of 2A3-26 positive DCs by flow cytometry}

After cell sorting, DCs were washed in PBS containing 1\% FCS and $0.01 \%$ sodium azide, and fixed in PBS containing $1 \%$ PFA for $20 \mathrm{~min}$ at $4{ }^{\circ} \mathrm{C}$. Then, DCs were washed in Perm/ Wash solution from the BD cytofix/CytopermTM Plus Kit (BD Biosciences). Intracellular amastigotes were stained with $5 \mu \mathrm{g} /$ $\mathrm{ml} 2 \mathrm{~A} 3-26 \mathrm{mAb}$ conjugated to Alexafluor 488 in Perm/Wash buffer for $30 \mathrm{~min}$ at $4{ }^{\circ} \mathrm{C}$ in the dark [1]. Then DCs were washed in Perm/Wash buffer and fixed with $1 \%$ PFA. Analysis was performed on the FACSAria.

\subsection{Microscopic observations of sorted DCs}

$D s R e d 2^{+} \mathrm{MHC}^{+}{ }^{+}$DCs were centrifuged on poly-Llysine-coated glass coverslips and incubated at $34^{\circ} \mathrm{C}$ for $30 \mathrm{~min}$. After thorough washing steps in PBS, cells were fixed with 4\% PFA and mounted on glass slides with Hoechst 33342-containing Mowiol. Incorporation of Hoechst into DNA allows the staining of both host-cell and amastigote nuclei. Epifluorescence microscopy images -mouse MHC II molecules in red, DC and Leishmania nuclei in blue- were acquired on an upright Zeiss Axioplan 2 microscope monitored by the Zeiss Axiovision 4.4 software.

\subsection{RNA integrity quality control}

Evaluation of RNA quality was performed by optical density measurement using the Nanodrop (Kisker, http://www. kisker-biotech.com) and by electrophoresis on a Lab-on-a-chip product using the Agilent 2100 Bioanalyzer (Agilent, http:// www.chem.agilent.com). RNA Integrity Number (RIN) scores were monitored for each sample providing an objective and standardised measure of RNA quality on a scale of 1-10 (the value 10 corresponding to the highest quality) $[18,19]$.

\subsection{Transcriptional analyses by $R T q P C R$ and Genechip analysis}

Total RNAs were extracted from biological triplicate DC cultures. For RTqPCR analyses, RNAs were reverse transcribed as previously described [20]. A SYBR Green-based real-time PCR assay (QuantiTect SYBR Green Kit, Qiagen) for quantitation of several mouse target genes was performed on a 384well plate LightCycler ${ }^{\circledR} 480$ system (Roche Diagnostics) Crossing Point values $(\mathrm{Cp})$ were determined by the second derivative maximum method of the LightCycler ${ }^{\circledR} 480$ Basic Software. Raw $\mathrm{Cp}$ values were input into qBase, a flexible and open source program for qPCR data management and analysis [21]. For normalisation calculations, 8 candidate control genes were tested (cycA, pbgd, h6pd, hprt, tbp, l19, gapdh, rpIIe) with geNorm [22] and Normfinder programs [23]. gapdh and rpIIe were selected as the most stable reference genes.

GeneChip hybridisations were performed following the Affymetrix two-cycle labelling protocol. QC assessment of Affymetrix recommended QC metrics was done using AffyGCQC program [24]. Affymetrix MIAME-compliant data have been made available through Gene Expression Omnibus databases (www.ncbi.nlm.nih.gov/projects/geo/, accession: GSE16644). Data processing, background correction, normalisation and signal quantification were carried out using GC-Robust Multiarray Analysis (GC-RMA) algorithm. Local pooled error tests [25] were performed to identify significant differences in gene expression between parasite-free and sorted parasite-harbouring DCs. Benjamini-Hochberg multipletest correction [26] was applied to control for the number of false positives (False Discovery Rate or FDR). We used GCRMA algorithm, LPE tests and Benjamini-Hochberg multiple-test correction as implemented in Partek ${ }^{\circledR}$ Genomics Suite $^{\mathrm{TM}}$ (Partek, St Louis, Missouri).

\subsection{Statistical analyses}

Two-sided Student's paired t-tests were used to compare data from flow cytometry experiments and gene expression studies performed on sorted samples $(6<n<13)$. A nonparametric Mann-Whitney bilateral U test was used for gene expression comparisons on unsorted samples $(n=5)$.

\section{Results}

\subsection{Principal features of DsRed2-expressing \\ L. amazonensis parasites}

Methods for sorting L. amazonensis-hosting DCs require the detection of intracellular amastigotes. Consequently, we 
generated transgenic LV79 L. amazonensis parasites that stably express the fluorescent molecule DsRed2 (DsRed2-LV79). These parasites displayed characteristics similar to wild type parasites (WT-LV79) such as the flow cytometric properties forward scatter-FSC and side scatter-SSC (morphological features) of both the promastigote developmental stage in vitro (data not shown) and the amastigote developmental stage (recovered from footpads of L. amazonensis-hosting nude mice) (Fig. 1A). The lesion onset and features were similar following the inoculation of the L. amazonensis-DsRed2 and WT-LV79parasites to BALB/c mice (Fig. 1B).
The orange fluorescence of DsRed2-LV79 amastigotes was detected both by epifluorescence microscopy and by flow cytometry (FC) with a $488 \mathrm{~nm}$ excitation wavelength (Fig. 1C). A key advantage of these fluorescent amastigotes was that neither cellular fixation nor permeabilisation was needed to efficiently detect them within the parasitophorous vacuole, allowing for the sorting of live DCs hosting live amastigotes. Indeed, the use of $D s$ Red2 fluorescence revealed only live amastigotes, since amastigotes killed by the leishmanicidal drug Leu-oMe [27] rapidly lost DsRed2 fluorescence (Fig. 1D, E).
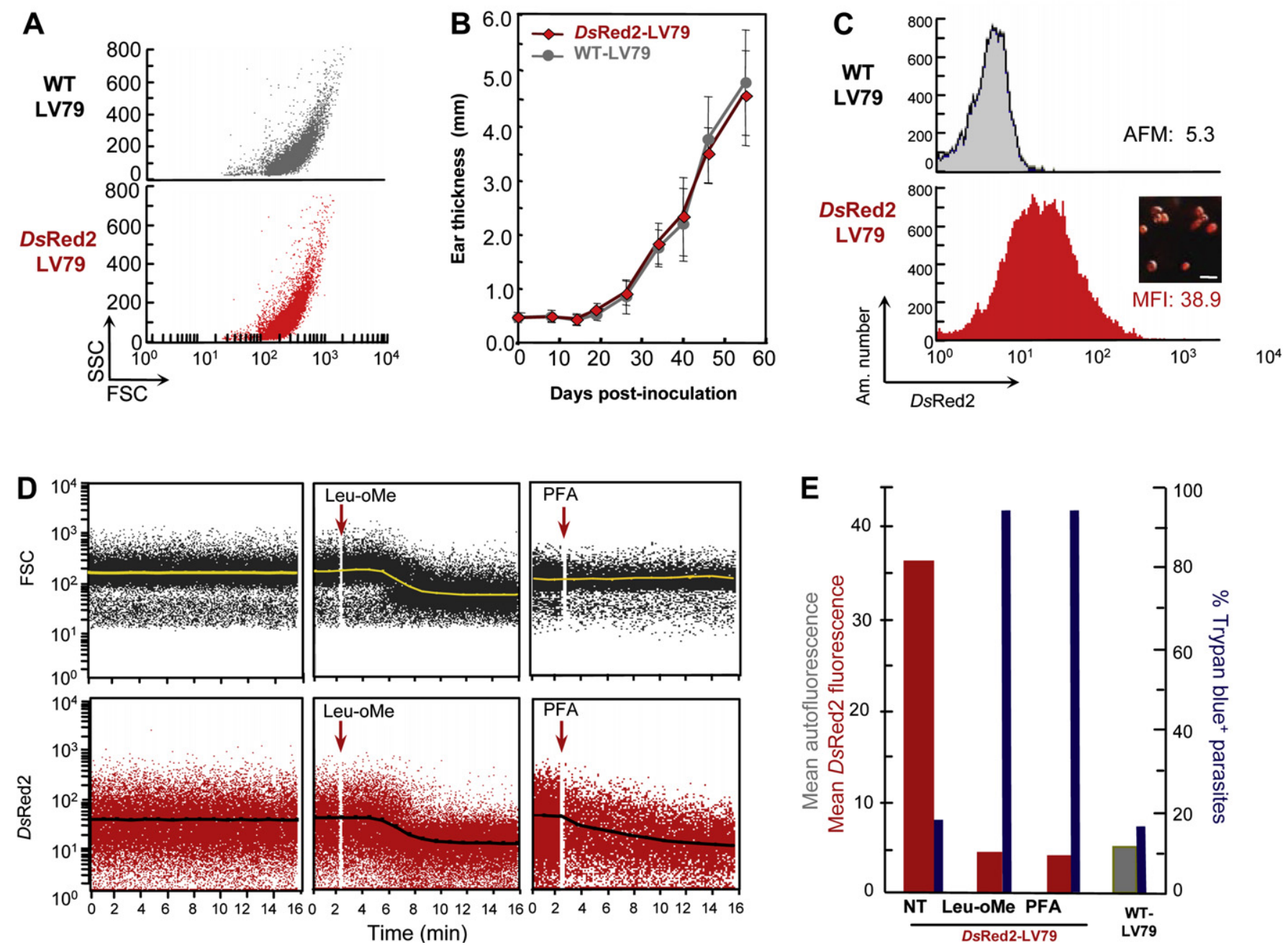

Fig. 1. Characteristics of L. amazonensis amastigotes expressing DsRed2. DsRed2-transgenic (DsRed2-LV79) and Wild type (WT-LV79) amastigotes were isolated from lesions of infected Swiss nude mice and further analysed by flow cytometry and epifluorescence microscopy. Analyses were performed without the addition of fixatives. (A) Flow cytometric analyses of forward (FSC) and side scatter (SSC) of ex vivo amastigotes post purification. (B) Ear clinical features (thickness) in BALB/c mice inoculated with WT-LV79 or DsRed2-LV79 amastigotes. Metacyclic WT- (grey line) or DsRed2-LV79 (red line) parasites (10,000) were inoculated into the ear dermis of $7 \mathrm{BALB} / \mathrm{c}$ mice $(n=14$ ears per group). Ear thickness was monitored every week for 8 weeks. Medians and standard deviations (SD) are indicated. (C) Detection of DsRed2 fluorescence from DsRed2-LV79 amastigotes. WT-LV79 autofluorescence (grey histograms) and DsRed2-LV79 orange fluorescence (red histogram) were analysed by cytofluorimetry. The mean of autofluorescence (AFM) and fluorescence intensity (MFI) are indicated. The $D s$ Red2 fluorescence of transgenic amastigotes was also detectable by epifluorescence microscopy (insert). Scale bar, $1 \mu \mathrm{m}$. (D, E) Impact of Leu-oMe or PFA exposure on DsRed2 fluorescence emission by isolated amastigotes (D) Real-time FC analyses of FSC (upper panels, black dots) and DsRed2 fluorescence (lower panels, red dots) of amastigotes exposed to $2 \mathrm{mM}$ Leu-oMe or $1 \%$ PFA. The addition of Leu-oMe or PFA is indicated by a red arrow. Lines correspond to real-time median values of FSC (upper panel) and DsRed2 fluorescence (lower panels). The leishmanicidal effect of Leu-oMe was correlated with a rapid loss of $D s$ Red2 fluorescence. (E) Impact of a $24 \mathrm{~h}$ exposure to Leu-oMe or PFA. The MFI of transgenic (red bars) and WT (grey bars; mean autofluorescence) amastigotes was measured by FC analysis and parasite death was assessed by trypan blue staining (blue histograms). 


\subsection{Setting up a reliable in vitro $D C$ culture for further characterisation of amastigote-hosting DCs}

DCs were derived in vitro from bone marrow progenitors of BALB/c mice [1]. In these cultures more than $95 \%$ of cells harboured the phenotype of myeloid DCs, and coexpressed CD11c, CD11a, CD11b and MHC class II molecules [1]. Only a minor fraction $(<5 \%)$ of the cultured cells did not express any MHC class II molecules (Fig. 2A) and did not display the morphology of DCs. DCs were cultured for $24 \mathrm{~h}$ in the absence or in the presence of freshly isolated DsRed2-LV79 amastigotes, this Leishmania developmental stage being efficiently phagocytosed by DCs [1]. DCs hosting DsRed2-LV79 amastigotes were easily detected via $D s$ Red2 fluorescence by both epifluorescence microscopy and flow cytometry (Fig. 2A, B, C). The percentage of infected cells and the mean DsRed2 fluorescence, both determined by flow cytometry analyses, varied with the parasite:DC ratio (Fig. 2A, B). First, we addressed the questions of the correlation between the percentages of the living DsRed2 ${ }^{+}$DCs and the intracellular presence of amastigotes, the latter being determined with the 2A3-26 $\mathrm{mAb}$, which has been shown to bind to the amastigote stage of L. amazonensis [1]. To this end, DCs were cultured with different amastigote:DC ratios (ranging from $0.5-8$ ) for $24 \mathrm{~h}$, fixed and permeabilised. Then, intracellular amastigotes were immunostained with the 2A3-26 mAb. The percentages of DsRed2-LV79-hosting DCs were highly correlated to the percentages of 2A3-26 positive DCs $\left(r^{2}=0.9736, p<0.001\right)$ (Fig. 2B). Consequently, FC analysis of DsRed2 fluorescence was a reliable approach to detect live DsRed2-LV79 amastigote-hosting DCs.
A

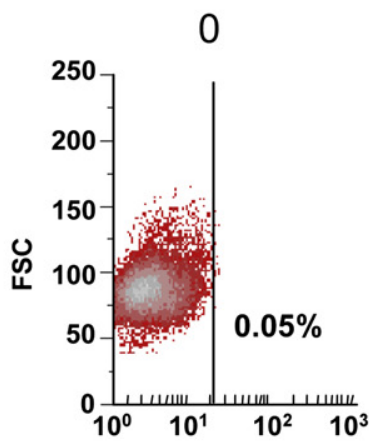

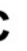

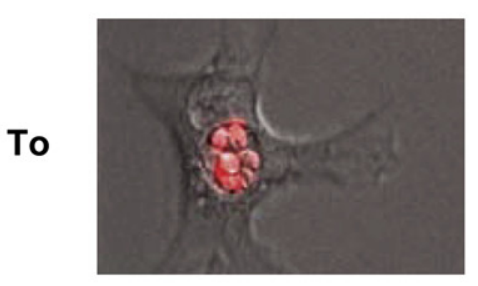

- Leu-oMe

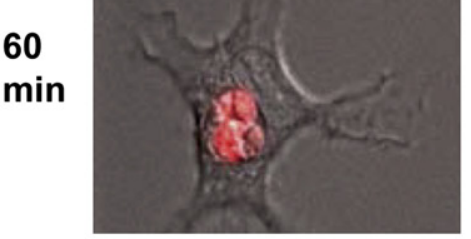

$2: 1$
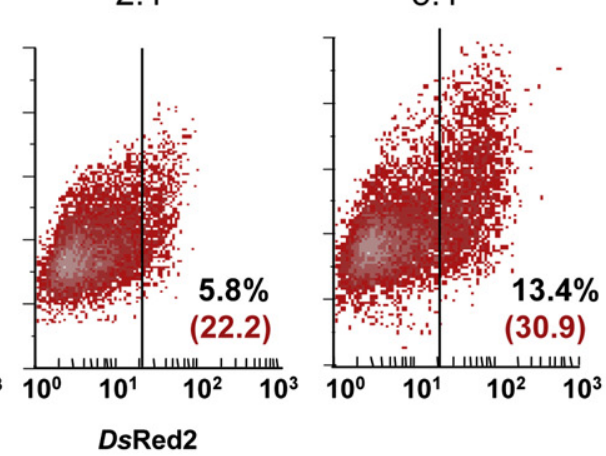
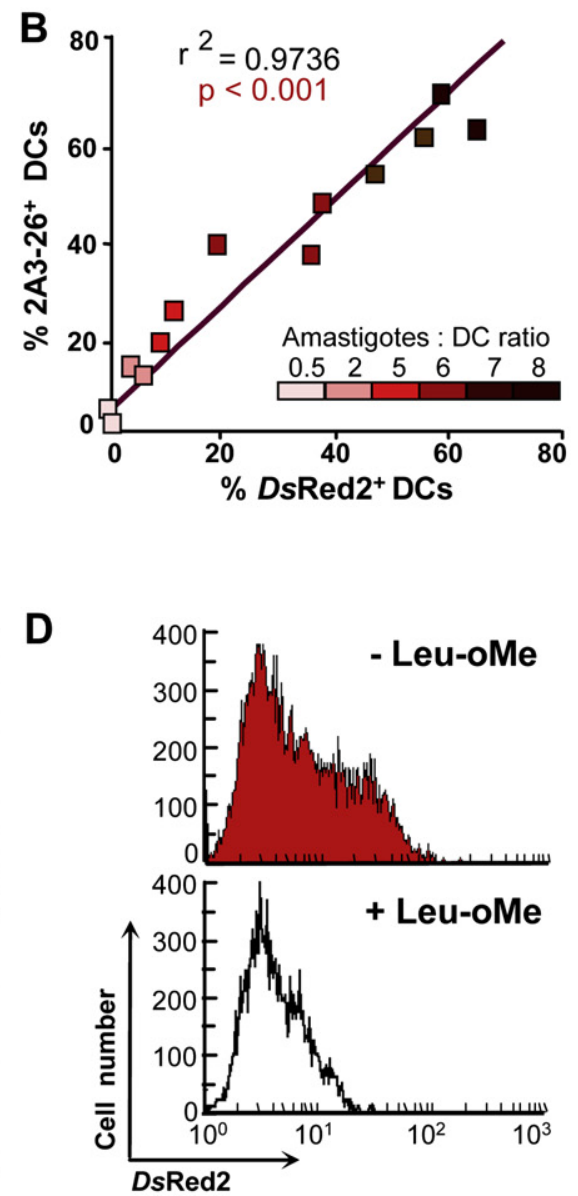

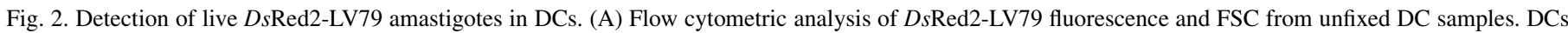

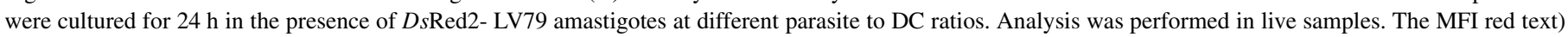

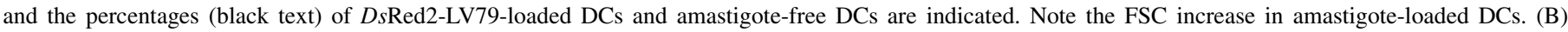

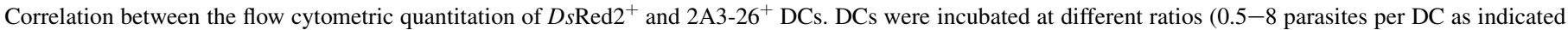

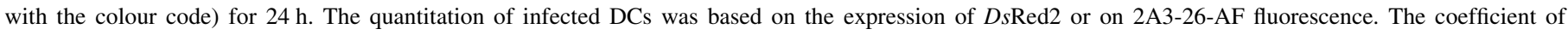

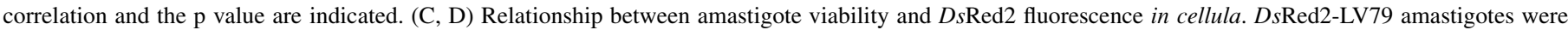

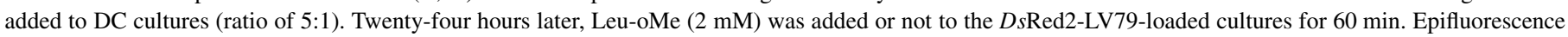

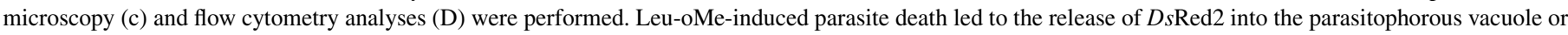

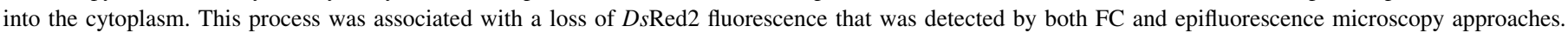


Secondly, we optimised the sorting procedure by setting up in vitro DC cultures with DCs hosting from one to four amastigotes. This intracellular amastigote number is similar to that observed in $\mathrm{CD} 11 \mathrm{c}^{+}$DCs collected from the lymph nodes of BALB/c mice 10 weeks after the inoculation of a low number of parasites into the ear dermis (Lang et al., unpublished data).

The "multiplicity of infection" of five was chosen for two reasons: i) the percentage of parasitized DCs is higher than with the 2:1 ratio (see Fig. 2A) ii) the percentage of $D s \operatorname{Red} 2^{+}$ cells reached $11.3 \% \pm 2.5 \%$ (one of eight representative experiments is displayed in Fig. 2B) and iii)withinthis $11.3 \%$ of live DCs hosting parasites more than $80 \%$ of DCs harboured one to four amastigotes (Supplementary Fig. 1).

Lastly, we wanted to ensure that only DCs hosting live amastigotes would be sorted. Thus, DsRred2 LV79-hosting DC cultures were exposed to the leishmanicidal drug Leu-oMe [27]. Not only there was a rapid diffusion of the fluorescence out of the amastigotes within parasitophorous vacuole, but there was also complete fluorescence extinction $60 \mathrm{~min}$ after the drug addition (Fig. $2 \mathrm{C}$ and D). These results indicated that DsRed2 fluorescence emission from Leishmania-infected DCs was only related to live amastigotes.

\subsection{High speed sorting of rare live DsRed2 amastigote- hosting DCs in BSL-2 containment}

The distribution of MHC class II molecules in DC cultures exposed to amastigotes was very similar to those observed in DCs left unexposed to the amastigotes (Fig. 3A1 and A2). The immunolabelling of surface MHC II molecules allowed us to exclude the low fraction of amastigote-hosting cells that did not express surface MHC II. The biparametric dot plot (Fig. 3A) was used to define the sorting gate on $\mathrm{MHC} \mathrm{II}^{+}$ $D s R e d 2^{+}$cells. The sorting was performed in samples exhibiting moderate $(9 \%)$ or low percentages $(0.5 \%$, Fig. $3 \mathrm{~A})$ of $\mathrm{MHC} \mathrm{II}^{+} D s \mathrm{Red} 2^{+}$cells. The risk of laboratory personnel exposure to aerosols during the cell sorting was minimised by the application of adequate BSL-2 measures (see "methods"). The Aerosol Management System added to the FACSAria cell sorter allowed for the vacuuming of aerosols into a special ultra-low penetrating air filter, and to generate an additional negative pressure in the sort collection chamber. The biosafety level 2 containment was performed by totally covering the cell sorter and the operator with a specialised biological safety cabinet (EP 20-16 hood), providing a localised negative pressure (see methods).

Due to the relative fragility of DCs, different experimental sorting conditions were tested, including variations on the pressure level ( 20 or $70 \mathrm{psi})$, the nozzle size $(100$ or $70 \mu \mathrm{m})$ and the flow rate ( 2 or 7 ) (Supplementary Fig. 2). Cell sorting procedures resulted in a moderate loss of viability in both control and infected cultures. The "70 $\mu \mathrm{m} / 70 \mathrm{psi} /$ flow rate of 7" combination resulted in a slight loss of viability, but permitted a higher rate of sorting, and thus shortened the sorting procedure and limited potential associated artefacts like DC activation/maturation. Consequently, this condition was applied for every cell sorting for infected DCs. The purification of sorted $D s \operatorname{Red} 2^{+}$DCs was first analysed by measuring parasite-specific RNAs in sorted and unsorted infected DC cultures (Supplementary Fig. 3). Firstly, all these transcripts were enriched in sorted samples. Secondly, these conditions enabled us to obtain pure sorted cells regardless of the initial percentage of amastigotes-hosting DCs present in the culture (9\% or $0.5 \%$ ) (Fig. 3A), as demonstrated by FC and microscopy analysis, and to select leucocytes that harboured at least two amastigotes (Fig. 3B). After every cell sorting, sequential decontaminating procedures were applied according to the conclusions drawn from Supplementary Fig. 4.

\subsection{Transcriptional signatures of live sorted \\ L. amazonensis-hosting DCs}

Since the preservation of RNA integrity was essential for our downstream investigations, electrophoresis on Agilent Lab-on-chips was applied to total RNA extracted from sorted and unsorted infected DC cultures. For all samples, RNA integrity numbers (RIN) were greater than 8 (Fig. 3C), and no differences could be detected between sorted and unsorted samples. These data revealed that RNA from sorted cells displayed the desired quality for Real-Time quantitative Polymerase Chain Reaction (RTqPCR) or Affymetrix studies.

We then verified that the cell sorting procedure on DC cultures exposed or not (control DCs) to amastigotes did not result in any DC maturation. To do so, as described under "Methods", a sort of the whole cell culture was performed according to FSC/SSC parameters and compared to unsorted samples. The cell sorting procedure did not modify the abundance of transcripts known to be modulated in DC activation, such as those coding for the chemokine-receptor 7 (CCR7), the membrane glycoprotein CD200, the DCspecific ICAM3-Grabbing Nonintegrin/CD209, the co-signalling molecules CD80, B7-H1, SLAMF1 and the chemokine-receptor 4 (CXCR4) (two-sided Student's paired ttest $p$-value $>1 \%$ with $6<n<13$; not shown). Then, the impact of loading with L. amazonensis was determined by measuring the abundance of RNA transcripts encoding CCR7, CD200, CD209, plus the Arg2 (arginase type II) and the arginase transporter Slc7a2 (Fig. 4A, B). As a positive control for the optimal induction of DC activation, cultures were exposed to live BCG for $24 \mathrm{~h}$ [1] (Fig. 4A). In unsorted cultures exposed to BCG, a strong and significant increase in transcript abundance was observed for the whole cell culture when compared to control DCs $(p<0.0001)$. In DCs exposed to DsRed2-LV79 amastigotes, no statistically significant differences in transcript abundance were observed when compared to control DCs (Fig. 4A). In contrast, the sorting of MHC class $\mathrm{II}^{+} D s R e d 2^{+}$DCs by flow cytometry revealed a significant modulation of transcripts that would not have been detected without the sorting procedure (Fig. 4B1). Indeed, a positive modulation was detected for CCR7 ( $p$-value $<0.01), \quad$ CD200 

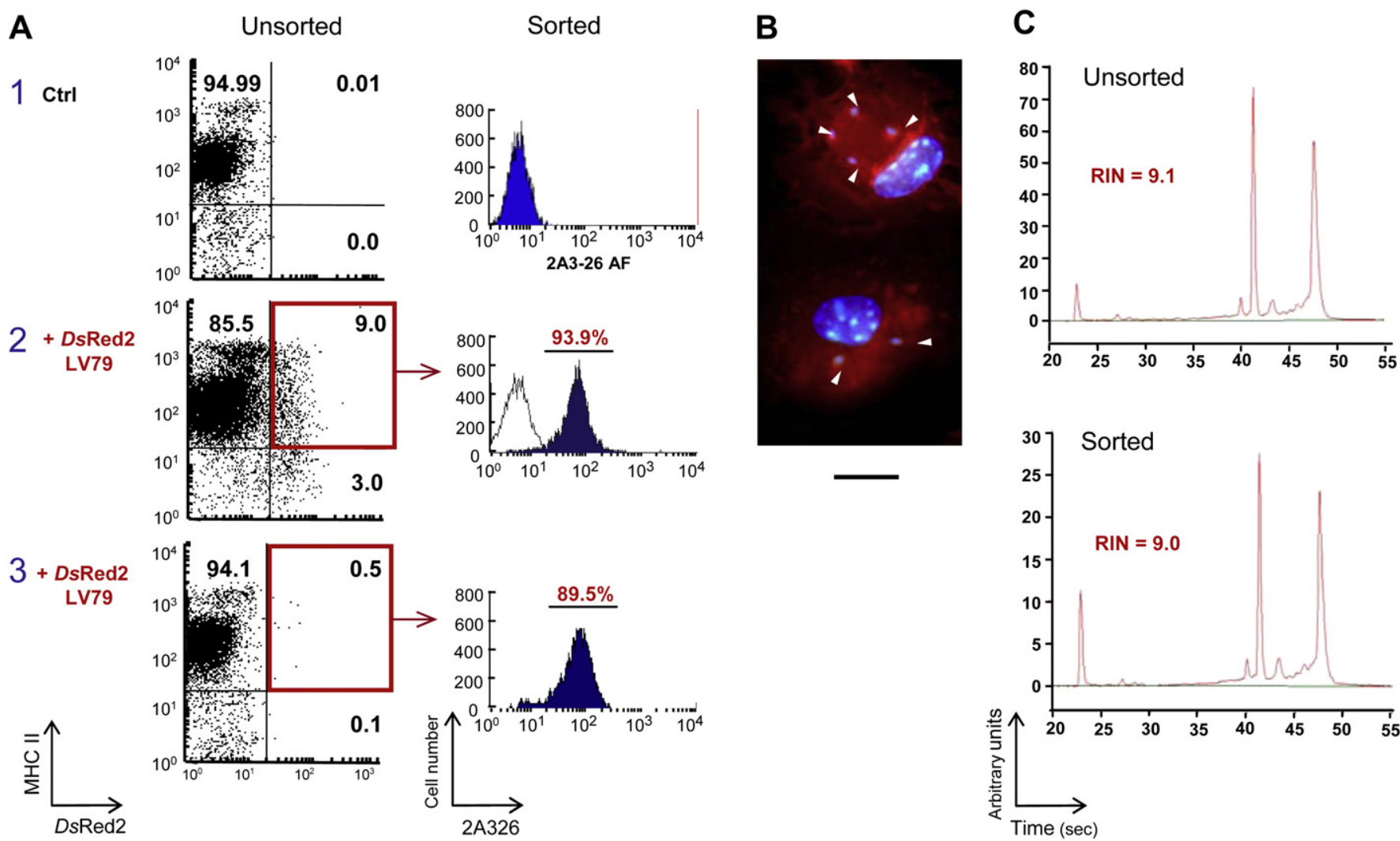

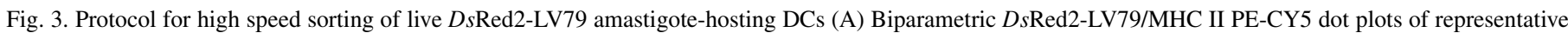

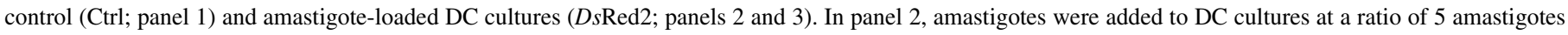

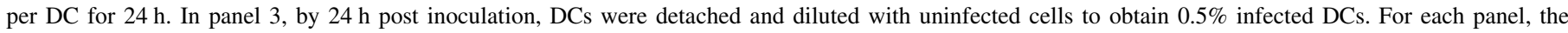

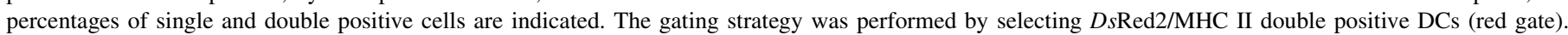

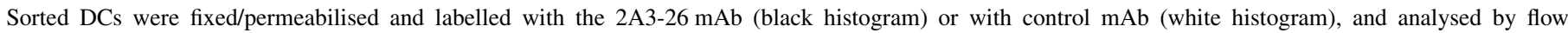

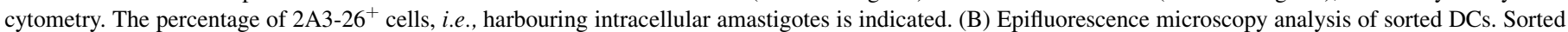

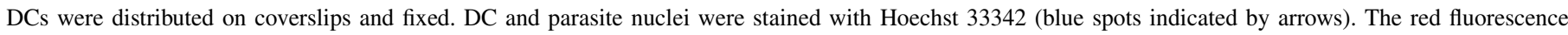

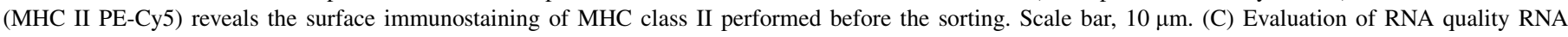
profiles from unsorted or sorted amastigote-loaded DCs were assessed by electrophoresis on Agilent Lab-on-Chips. RIN values are indicated.

$(p<0.05)$, Slc7a2 $(p<0.1)$, and $\operatorname{Arg} 2(p<0.05)$, (twosided Student's paired t-test with $6<n<9)$. In addition, a negative modulation was observed for CD209 $(p<0.01)$. Therefore, a genome-wide transcriptional analysis was performed by comparing the gene expression profiles of control DCs and amastigote-hosting DCs using the Affymetrix technology. Among the 45,101 probe-sets of the Mouse430_2 GeneChip, $613(1.4 \%)$ were captured with a differential expression at the 5\% significance level (Fig. 4B2). Comparable fold change values were obtained between the Affymetrix technology and the RTqPCR method. Among these probe-sets, 470 represented genes with a known function. Compared analysis pinpointed the modulation of various genes involved in the L-arginine and polyamine pathways (Fig. 4B3) that would not have been detected in unsorted cells. Indeed, modulation of transcripts encoding the arginine transporter Slc7a2 and Arg2 were revealed (mean $\mathrm{FC}+5.63$ and +4.2 ) in sorted Leishmaniahosted DCs. This analysis also revealed the weak modulation of genes involved in the polyamine pathway such as Sat1 (spermidine/spermine N1-acetyltransferase 1; FC + 2.16).

\section{Discussion}

In the present study we describe a reliable and safe method for sorting live DCs hosting L. amazonensis. Taking into consideration both scientific issues, including the rarity of live amastigotes-harbouring DCs in the mouse tissues hosting Leishmania, and ethical issues, such as avoiding the sacrifice of numerous mice, we designed the amastigotehosting DC sorting procedure with model DC populations generated and mastered in vitro. To this end, we describe a high speed sorting procedure performed in a biosafety level 2 containment $[10,11]$, that allows for the purification of rare amastigote-hosting DCs and accurate monitoring of DC features. The use of L. amazonensis transgenic parasites expressing the DsRed2 fluorescent protein, which revealed only live amastigotes, allowed us to sort live $D s \operatorname{Red} 2 \mathrm{MHC}$ class II positive DCs that represented only $0.5 \%$ of total cells, a percentage close to the one found in tissues of Leishmania-infected mice [28]. A major advantage of this strategy is the ability to sort live cells, the DC death being always a rare outcome. This strategy also allows for the isolation of a high quality RNA from the sorted cells, which 
A
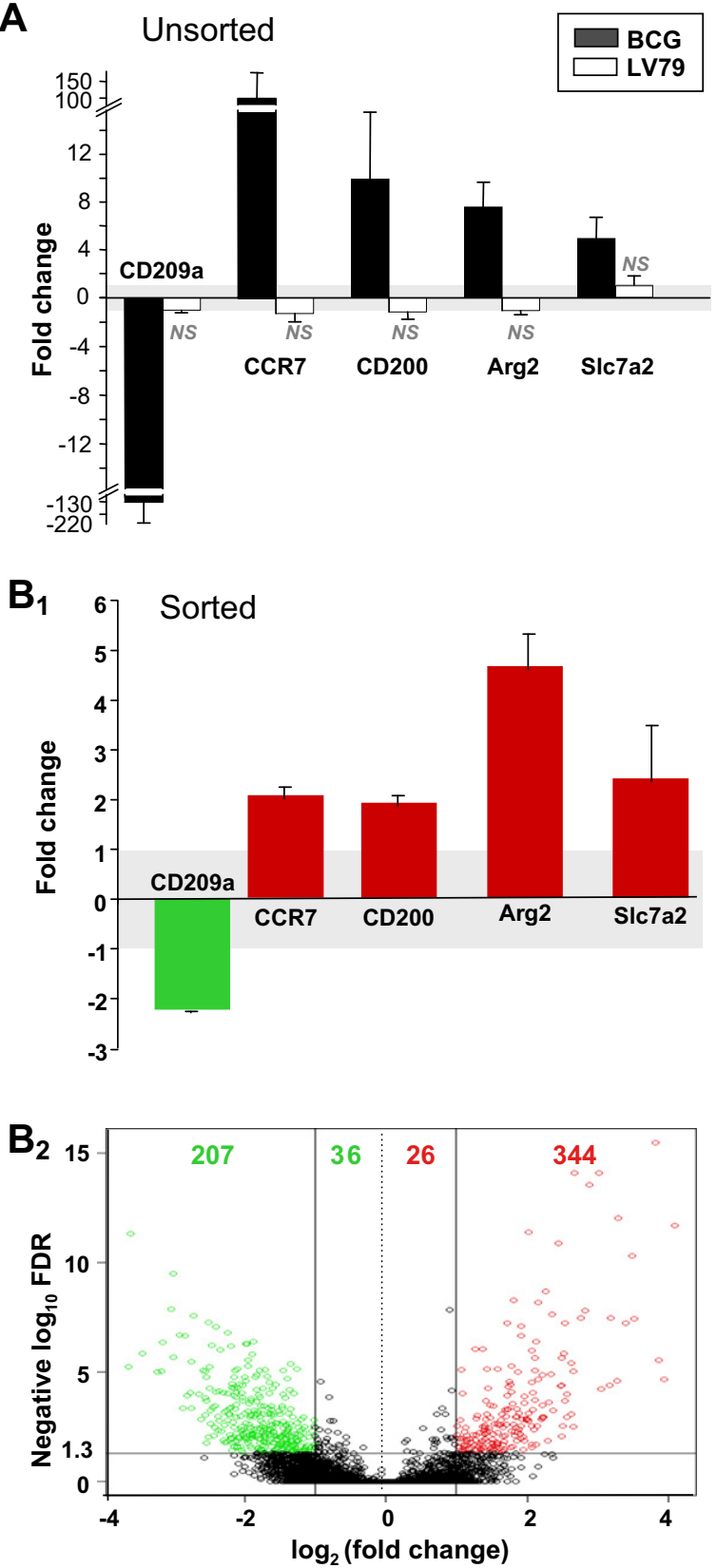

\begin{tabular}{cllll} 
B 3 Gene & Probe set & log2 FC & FC & FDR \\
& & & & \\
\hline \multirow{2}{*}{ SIc7a2 } & 1422648_at & 2.626 & 6.15 & $3.8 \mathrm{E}-06$ \\
& 1426008_a_at & 2.56 & 5.90 & 0.0011 \\
& 1436555_at & 2.53 & 5.78 & 0.0002 \\
& 1450703_at & 2.23 & 4.69 & 0.0011 \\
\hline \multirow{2}{*}{ arg2 } & 1438841_s_at & 2.09 & 4.26 & 0.0042 \\
& 1418847_at & 2.08 & 4.23 & 0.0005 \\
\hline \multirow{2}{*}{ Sat1 } & 1420502_at & 1.11 & 2.16 & 0.0052
\end{tabular}

Fig. 4. Differential transcript analysis of sorted MHC II positive DCs harbouring or not DsRed2-LV79 (A) Transcript analysis of unsorted DC cultures DsRed2-LV79 (5 per DC) or BCG (10 per DC) were added to DCs for $24 \mathrm{~h}$. RNA extraction, reverse transcription and RTqPCR were performed can then be used for different purposes, including gene expression analyses with real-time PCR and oligonucleotide microarray hybridisations. This new approach revealed for the first time a diverse expression pattern in transcripts from sorted live amastigote-hosting DCs that would have been undetectable in unsorted samples. As a relevant biological feature of the amastigote-hosting DCs, we identified a significant up-regulation of transcripts coding for molecules involved in L-arginine metabolism, which is known to be induced as either a source of $\mathrm{NO}$ or as a source of polyamines [29,30]. The significant and coordinated up-regulation of an L-arginine transporter, arginase2, and spermidine/spermine N1-acetyltransferase 1/Sat1 [31], indicates that arginine catabolism for the polyamine generation is dominating over L-arginine catabolism for NO generation. It would be interesting to follow the modulation of these metabolic pathways at different time points post L. amazonensis intradermal inoculation to mice. Indeed, within the dermis loaded with intracellular amastigotes, DCs could display phenotypes ranging from NO to polyamine synthesis. The recent study performed in C57BL/ 6 mice suggests that the presence of iNOS-producing inflammatory DCs in the Leishmania majorloaded footpads was closely associated with the parasite load reduction phase, the latter assessing the dominance of a type 1 environment in the L. major-loaded footpad [32].

The technique developed herein for in vitro DC samples will enable many new applications by taking advantages of polychromatic flow cytometry analyses. Indeed, it would allow for the specific sorting of DC subsets according to the simultaneous expression of surface molecules (CD11c, CD80, CD86, chemokine-receptors, anti-apoptotic signalling receptors....). In addition, the settings and sorting conditions that we defined here constitute useful parameters for the further sorting of amastigote-hosting DCs from mouse biological samples such as the ears and the ear-draining lymph nodes.

on unsorted DC cultures. Displayed data are the mean fold changes for Arg2, CCR7, CD200, CD209 and Slc7a2 transcripts between DCs in contact with BCG or amastigotes and control DCs $(n=5$ experiments). For all target genes under study there is a statistically significant difference between control DCs and DCs with BCG ( $p=<0.001$; nonparametric Mann-Whitney bilateral U test) but not between control DCs and DCs in contact with Leishmania amastigotes (NS = non significant). (B) Transcript analysis of sorted DCs harbouring DsRed2-LV79. Analyses were performed by RTqPCR (b1) and Genechip analysis (Affymetrix) (b2, b3). (B1) Bar charts of a representative experiment showing the fold change values of 5 transcripts between $D s$ Red2-housing sorted DCs (DsRed2/MHC II positive cells) and sorted control DCs (MHC II positive cells). (B2) Volcano plot representation of the differential gene expression between sorted L. amazonensis housing-mouse DCs and non parasitised DCs. Modulated probesets with False Discovery Rate (FDR) $<0.05$ are above the horizontal line. The number of modulated probe-sets is indicated. A total of 613 probe-sets show significant differential expression. Up-regulated probe-sets with a fold change $>2\left[\log _{2}\right.$ (fold change) $\left.>1\right]$ are on the right side of the right vertical line, while down-regulated probe-sets (207) with a fold change $<-2$ are on the left side of the left vertical line. A total of 62 probe-sets showed differential expression with a fold change between -2 and +2 . (B3) List of DC transcripts related to L-arginine metabolism and the polyamine pathway regulated in sorted infected DCs. 


\section{Acknowledgments}

We particularly acknowledge Marie Nguyen-de-Bernon for her expertise in flow cytometry. Activities presented in this work are funded i) the Institut Pasteur, ii) the Fonds Dédié Sanofi-Aventis / Ministère de l'Enseignement Supérieur de la Recherche «Combattre les Maladies Parasitaires» and by the European Communities (EC), grant agreement n²23414".

\section{Appendix. Supplementary material}

Supplementary material associated with this article can be found in the online version at doi:10.1016/j.micinf.2009.09. 014.

\section{References}

[1] E. Prina, S.Z. Abdi, M. Lebastard, E. Perret, N. Winter, J.C. Antoine, Dendritic cells as host cells for the promastigote and amastigote stages of Leishmania amazonensis: the role of opsonins in parasite uptake and dendritic cell maturation. J. Cell Sci. 117 (2004) 315-325.

[2] L.G. Ng, A. Hsu, M.A. Mandell, B. Roediger, C. Hoeller, P. Mrass, Migratory dermal dendritic cells act as rapid sensors of protozoan parasites. PLoS Pathog 4 (2008) e1000222.

[3] N.C. Peters, J.G. Egen, N. Secundino, A. Debrabant, N. Kimblin, S. Kamhawi, et al., In vivo imaging reveals an essential role for neutrophils in leishmaniasis transmitted by sand flies. Science 321 (2008) 970-974.

[4] A.C. Misslitz, K. Bonhagen, D. Harbecke, C. Lippuner, T. Kamradt, T. Aebischer, Two waves of antigen-containing dendritic cells in vivo in experimental Leishmania major infection. Eur. J. Immunol. 34 (2004) $715-725$.

[5] A. Kissenpfennig, S. Henri, B. Dubois, C. Laplace-Builhe, P. Perrin, N. Romani, et al., Dynamics and function of Langerhans cells in vivo: dermal dendritic cells colonize lymph node areas distinct from slower migrating Langerhans cells. Immunity 22 (2005) 643-654.

[6] L. Xin, K. Li, L. Soong, Down-regulation of dendritic cell signaling pathways by Leishmania amazonensis amastigotes. Mol. Immunol. 45 (2008) 3371-3382.

[7] C. Auffray, M.H. Sieweke, F. Geissmann, Blood monocytes: development, heterogeneity, and relationship with dendritic cells. Annu. Rev. Immunol. 27 (2009) 669-692.

[8] Y. Xu, Y. Zhan, A.M. Lew, S.H. Naik, M.H. Kershaw, Differential development of murine dendritic cells by GM-CSF versus Flt3 ligand has implications for inflammation and trafficking. J. Immunol. 179 (2007) 7577-7584.

[9] B.J. Bevis, B.S. Glick, Rapidly maturing variants of the Discosoma red fluorescent protein (DsRed). Nat. Biotechnol. 20 (2000) 83-87.

[10] I. Schmid, C. Lambert, D. Ambrozak, G.E. Marti, D.M. Moss, S.P. Perfetto, International society for analytical cytology biosafety standard for sorting of unfixed cells. Cytometry Part A 71A (2007) 414-437.

[11] I. Schmid, J.K.A. Nicholson, J.V. Giorgi, G. Janossy, A. Kunkl, P.A. Lopez, et al., Biosafety guidelines for sorting of unfixed cells. Cytometry 28 (1997) 99-117.

[12] S. Goyard, H. Segawa, J. Gordon, M. Showalter, R. Duncan, S.J. Turco, et al., An in vitro system for developmental and genetic studies of Leishmania donovani phosphoglycans. Mol. Biochem. Parasitol. 130 (2003) 31-42.

[13] M.C. Bosetto, S. Giorgio, Leishmania amazonensis: multiple receptorligand interactions are involved in amastigote infection of human dendritic cells. Exp. Parasitol. 116 (2007) 306-310.
[14] G.F. Späth, S.M. Beverley, A lipophosphoglycan-independent method for isolation of infective Leishmania metacyclic promastigotes by density gradient centrifugation. Exp. Parasitol. 99 (2001) 97-103.

[15] H. Lecoeur, A. Langonne, L. Baux, D. Rebouillat, P. Rustin, M.C. Prevost, et al., Real-time flow cytometry analysis of permeability transition in isolated mitochondria. Exp. Cell Res. 294 (2004) 106-117.

[16] I. Méderlé, I. Bourguin, D. Ensergueix, E. Badell, J. Moniz-Peireira, B. Gicquel, et al., Plasmidic versus insertional cloning of heterologous genes in mycobacterium bovis BCG: impact on in vivo antigen persistence and immune responses. Infect. Immun. 70 (2002) 303-314.

[17] T. Zal, A. Volkmann, B. Stockinger, Mechanisms of tolerance induction in major histocompatibility complex class ii-restricted t-cells specific for a blood-borne self-antigen. J. Exp. Med. 180 (1994) 2089-2099.

[18] A. Schroeder, O. Mueller, S. Stocker, R. Salowsky, M. Leiber, M. Gassmann, et al., The RIN: an RNA integrity number for assigning integrity values to RNA measurements. BMC. Mol. Biol. 7 (2006) -.

[19] S. Fleige, V. Walf, S. Huch, C. Prgomet, J. Sehm, M.W. Pfaffl, Comparison of relative mRNA quantification models and the impact of RNA integrity in quantitative real-time RT-PCR. Biotechnol. Lett. 28 (2006) 1601-1613.

[20] O. Wagner-Ballon, H. Chagraoui, E. Prina, M. Tulliez, G. Milon, H. Raslova, et al., Monocyte/macrophage dysfunctions do not impair the promotion of myelofibrosis by high levels of thrombopoietin. J. Immunol. 176 (2006) 6425-6433.

[21] J. Hellemans, G. Mortier, A. De Paepe, F. Speleman, J. Vandesompele, qBase relative quantification framework and software for management and automated analysis of real-time quantitative PCR data. Genome Biol 8 (2007) R19.

[22] J. Vandesompele, K. De Preter, F. Pattyn, B. Poppe, N. Van Roy, A. De Paepe, et al., Accurate normalization of real-time quantitative RT-PCR data by geometric averaging of multiple internal control genes. Genome Biol. 18 (2002).

[23] C.L. Andersen, J.L. Jensen, T.F. Orntoft, Normalization of real-time quantitative reverse transcription-PCR data: a model-based variance estimation approach to identify genes suited for normalization, applied to bladder and colon cancer data sets. Cancer Res. 64 (2004) 5245-5250.

[24] J. Osorio y Fortea, E. Prina, T. Lang, G. Milon, C. Davory, J.Y. Coppée, et al., Affycqc: a web interface to detect outlying genechips with extreme studentized deviate tests. J. Bioinform. Comput. Biol. 6 (2008) 317-334.

[25] N. Jain, J. Thatte, T. Braciale, K. Ley, M. O'Connell, J.K. Lee, Localpooled-error test for identifying differentially expressed genes with a small number of replicated microarrays. Bioinformatics 19 (2003) $1945-1951$.

[26] Y. Benjamini, Y. Hochberg, Controlling the false discovery rate a practical and powerful approach to multiple testing. J. R. Stat. Soc., Ser. B, Stat. Methodol. 19 (1995) 289-300.

[27] E. Prina, E. Roux, D. Mattei, G. Milon, Leishmania DNA is rapidly degraded following parasite death: an analysis by microscopy and realtime PCR. Microbes Infect 9 (2007) 1307-1315.

[28] B. Leon, M. Lopez-Bravo, C. Ardavin, Monocyte-derived dendritic cells formed at the infection site control the induction of protective Thelper 1 responses against Leishmania. Immunity 26 (2007) 519-531.

[29] T. Gotoh, M. Mori, Arginase II downregulates nitric oxide (NO) production and prevents NO-mediated apoptosis in murine macrophagederived RAW 264.7 cells. J. Cell Biol. 144 (1999) 427-434.

[30] S.C. Roberts, M.J. Tancer, M.R. Polinsky, K.M. Gibson, O. Heby, B. Ullman, Arginase plays a pivotal role in polyamine precursor metabolism in Leishmania. Characterization of gene deletion mutants. J. Biol. Chem. 279 (2004) 23668-23678.

[31] A.E. Pegg, Spermidine/spermine-N(1)-acetyltransferase: a key metabolic regulator. Am J Physiol Endocrinol Metab 294 (2008) E995-1010.

[32] C. De Trez, S. Magez, S. Akira, B. Ryffel, Y. Carlier, E. Muraille, iNOSproducing inflammatory dendritic cells constitute the major infected cell type during the chronic Leishmania major infection phase of C57BL/6 resistant mice. PLoS Pathog 5 (2009) e1000494. 